\title{
Analysis of Postural Stability in a Female-Patient with Parkinson's Disease Before and After Double-Sided Dbs Implantation-A 2-Year Case-Study
}

\author{
Elżbieta Mirek $^{1^{\star}}$, Magdalena Filip ${ }^{1}$ and Michał Michalski ${ }^{2}$ \\ ${ }^{1}$ University School of Physical Education in Cracow, Poland \\ ${ }^{2}$ Department of Neurology and Neurorehabilitation, John Paul's II Hospital, Cracow, Poland \\ *Corresponsing author: Elżbieta Mirek, University School of Physical Education in Cracow, Poland, Tel: +48 695327424 ; Fax: +48 695327 ; E-mail: mirek.ela@wp.pl
}

Rec Date: May 23, 2016; Acc Date: June 21, 2016; Pub Date: June 27, 2016

Copyright: (c) 2016 Mirek E, et al. This is an open-access article distributed under the terms of the Creative Commons Attribution License, which permits unrestricted use, distribution, and reproduction in any medium, provided the original author and source are credited.

\begin{abstract}
Parkinson's disease, PD is one of the most frequent, progressive neurodegenerative diseases of the central nervous system. The most important four core symptoms of PD include: rigidity, resting tremor, bradykinesia, and postural instability. In addition to pharmacological treatment of PD, also available are advanced methods such as infusion therapies and deep brain stimulation (DBS). The aim of the undertaken study for 69-year-old female patient diagnosed with PD is the preliminary assessment of the use of a balance platform as an objective tool for monitoring the progression of imbalances in the course of PD. Due to the increase of PD symptoms (resting and kinetic limb tremors, drug-induced dyskinesias, paranoid delusions with hallucinations) and poor tolerance of the drugs, she was approved for double-sided DBS implantation. An increase in postural sway in both the sagittal and frontal planes was observed in patients after direct double-sided DBS implantation and pharmacological treatment. During the sixmonth observation period after combined DBS and physiotherapy, improvement occurred in the analyzed parameters. Computer testing with the usage of the balance platform is a repeatable method that can be successfully applied in the assessment of movement disorders and the analysis of the results of pharmacological treatment, DBS or the physiotherapy of patients with PD.
\end{abstract}

\section{Introduction}

Parkinson's disease (PD) is one of the most frequent and progressive neurodegenerative diseases of the central nervous system. In Poland, there are about 80 thousand registered people diagnosed with PD. The most important four-core symptoms of PD include: rigidity, resting tremors, bradykinesia, and postural instability. With progression of the disease, there are also cognitive disorders [1,2]. Postural instability in $\mathrm{PD}$ is directly linked to an increased risk of falls and consequently, loss of independence [3]. One of the most commonly used methods for evaluating balance control is analysis of horizontal sway of the body as a function of time by measuring the movements of the point of application of the resultant reaction force of the ground - center of pressure (COP). In the frontal plane, these forces are activated mainly through the action of adductor and abductor muscles of the hip joints and the muscles responsible for ankle-joint inversion and eversion. However, in the sagittal plane, they are activated through three-headed calf muscle activity, tibialis anterior within the ankle-joint, hip quadriceps and gluteus maximus. Proper anti-gravity muscle activity responsible for vertical posture (sagittal plane) is a prerequisite for safe and economic locomotion [4,5]. Clinical and posturographic research have significantly contributed to explaining the complex pathophysiology of postural instability in patients with PD. Still unexplained remain posture disturbances, partly because of the difficulty in distinguishing the disease process and compensation mechanisms, and also due to the fact that there are no standard techniques measuring balance or postural instability [6-8]. In addition to pharmacological treatment of $\mathrm{PD}$, advanced methods such as infusion therapies and deep brain stimulation (DBS) are also available [9]. DBS is an operating procedure involving implantation of electrodes, usually around the area of the subthalamic nucleus or globus pallidus. Then they are connected using a cable with a neurostimulator, which is stitched to the collar bone area. This results in electrical stimulation of the brain regions responsible for the control of movement, as well as blocking abnormal nerve signals causing PD symptoms [10]. Research confirms that the greatest resting tremors are most greatly reduced by stimulation of the bilateral subthalamic nucleus. This effect may be maintained for up to 5 years [11]. The aim of the undertaken study for a person with PD is the preliminary assessment of the use of a balance platform as an objective tool for monitoring the progression of imbalances in the course of PD.

\section{Patient Description}

The study involved a 69-year-old female patient diagnosed with PD in 2012. The patient with stage 3 of the disease according to Hohen and Yahr, UPDRS Part 365 points, "off” phase. Due to the increase of PD symptoms (resting and kinetic limb tremors, drug-induced dyskinesias, paranoid delusions with hallucinations) and poor tolerance of the drugs, she was approved for DBS of the bilateral subthalamic nucleus in April 2014 (1500 mg levodopa, 6 mg biperiden, $300 \mathrm{mg}$ amantadine, $4 \mathrm{mg}$ Selegiline). 7 months after the qualifications, the first implantation of electrodes into the right hemisphere of the brain was carried out, parameters were set and pharmacological treatment was adjusted (after implantation of DBS reducing dopaminergic therapy was applied in doses of $250 \mathrm{mg}$ levodopa, 100 $\mathrm{mg}$ amantadine, $2 \mathrm{mg}$ biperiden). One month later, the procedure was repeated for the other hemisphere of the brain (drug treatment only $200 \mathrm{mg}$ levodopa). Before qualifying for DBS, the patient was assessed on the BIODEX platform during the "on" phase. Further tests were performed after the first and second implantations. The last test was conducted in May 2016. At that time, the patient was not undergoing any pharmacological treatment (UPDRS part 335 points, "off" phase). Postural stability was evaluated (the average score out of 3 trials, each 
Page 2 of 3

lasting $20 \mathrm{sec}$., stable platform). The analyzed parameter during free standing was the center of pressure (COP). The test always took place in the same conditions and according to the same Biodex Balance System SD protocol. Throughout the whole study, the patient had no orthopedic ailments (independent movement), cognitive disorders or other diseases that could have significantly affected postural stability. Between the 3rd and 4th tests, the patient was recommended to walk for $30 \mathrm{~min}$, three times a week. The patient gave her consent to participate in the study and provided her medical history and test results.

\section{Results}

The results of the tests on the balance platform are presented in (Table 1).

\begin{tabular}{|c|c|c|c|c|c|c|c|}
\hline \multirow[t]{2}{*}{ No. } & \multirow[t]{2}{*}{ Date } & \multicolumn{6}{|l|}{ Postural stability } \\
\hline & & $\begin{array}{l}\text { Overall } \\
\text { Postural Sway } \\
\text { Index }\left[^{\circ}\right]\end{array}$ & SD & $\begin{array}{l}\text { A/P Postural } \\
\text { Sway Index }\left[\left[^{\circ}\right]\right.\end{array}$ & SD & $\begin{array}{l}\text { M/L Postural } \\
\text { Sway Index }\left[{ }^{\circ}\right]\end{array}$ & SD \\
\hline 1 & Apr-14 & 4.6 & 4.6 & 2.2 & 1.3 & 3.9 & 1.22 \\
\hline 2 & Oct-14 & 6.4 & 6.4 & 5.8 & 2.04 & 2.3 & 1.86 \\
\hline 3 & Nov-14 & 8.2 & 8.2 & 6.0 & 0.96 & 5.5 & 0.55 \\
\hline 4 & May-16 & 5.6 & 5.6 & 5.2 & 1.3 & 1.9 & 1.42 \\
\hline $\begin{array}{l}\mathrm{SD}- \\
\mathrm{A} / \mathrm{P}- \\
\mathrm{M} / \mathrm{L}-\end{array}$ & $\begin{array}{l}\text { ition } \\
\text { gittal plane } \\
\text { (frontal ple }\end{array}$ & & & & & & \\
\hline
\end{tabular}

Between the first and last test, an increase in Postural Sway Index from $2.2 \pm 1.3^{\circ}$ to $5.2 \pm 1.61^{\circ}$ was observed in the sagittal plane, while in the coronal plane, there was a decrease in Postural Sway Index from $3.9 \pm 1.22^{\circ}$ to $1.9 \pm 1.42^{\circ}$. Simultaneously, there was a slight increase in Overall Postural Sway Index from $4.6 \pm 1.34^{\circ}$ to $5.6 \pm 1.61^{\circ}$. A significant increase in all analyzed parameters was observed in the study immediately after double-sided DBS implantation. The largest deterioration was observed in the M/L postural Sway index, which increased from $3.9 \pm 1.22^{\circ}$ to $5.5 \pm 0.55^{\circ}$.

\section{Discussion}

Despite the degenerative nature and burdensome symptoms of Parkinson's disease, the use of pharmacological treatment and rehabilitation allows patients' well-functioning and active participation in everyday life. The symptoms associated with Parkinson's disease and its duration have a significant effect on postural control disorders $[12,13]$. Analysis of postural stability of the female-patient in the first study clearly shows irregularities manifested mainly by a particularly high indicator in the $\mathrm{M} / \mathrm{L}$ plane equaling $3.9 \pm 1.22^{\circ}$, while in the sagittal plane, the indicator totals $2.2 \pm 1.3^{\circ}$. This proves the insufficient work of antigravity muscles, which directly translates to gait disturbances and falls. Comparing the patient's first and last test, it can be seen that after 1.5 years of using DBS therapy and physiotherapy, the patient achieved a lower stability indicator in the $\mathrm{M} / \mathrm{L}$ plane with a simultaneous increase in the indicator value of stability in the A/P plane. At the same time, there was a slight increase in the overall stability rate. This reflects improvement in the functioning of antigravity muscles, as well as better control of postural sway in the frontal plane despite the progressive, neurodegenerative nature of the disease. During normal, proper standing position, the range of postural sway in the sagittal plane should be two to three times higher than in the frontal plane [5]. These proper values of postural sway were achieved in the patient after using a combination of DBS and physiotherapy.

The presented case study allows to analyze the impact of DBS on postural stability of the patient. The results showed significant deterioration of postural stability in both the sagittal and frontal planes immediately after DBS implantation. Research conducted by Rocchi analyzing postural stability in 6 people with PD and an 11-person control group shows that people with PD have impaired postural sway in stance. Moreover, the researchers found that treatment with levodopa increases postural sway abnormalities, whereas treatment with deep brain stimulation improves postural sway [14]. This is in agreement with the analysis presented in this case study. The patient, during the usage of only pharmacological treatment and pharmacological treatment combined with DBS, received the highest $\mathrm{M} / \mathrm{L}$ postural sway. Research on a larger group of people is recommended. The Deep Brain Stmulation Study Group presented research conducted among 134 patients with PD following doublesided DBS implantation using the UPDRS [15]. Patients achieved significant improvement in motor function. A year and a half after double-sided DBS implantation, the patient received 35 points in the UPDRS Part 3, which is reflected in improve postural sway.

The use of double-sided DBS in PD results in a decrease in resting tremors, dyskinesia and muscle tension [11]. As a result, the most manifested symptom becomes postural instability. In our study, the patient received the highest score of $\mathrm{M} / \mathrm{L}$ postural sway immediately after the combined application of DBS therapy and pharmacological treatment.

Posturography is an objective research tool that allows the analysis of postural sway in both the sagittal and frontal planes [16]. Looking through the various studies, it may be noted that the assessment of balance with a balance platform is very popular, because it is a tool that is easy to use, gives quantitative results and explores the greatly precise activity of the balance of a human. Scientists have proven the 
Citation: Mirek E, Filip M, Michalski M (2016) Analysis of Postural Stability in a Female-Patient with Parkinson's Disease Before and After Double-Sided Dbs Implantation-A 2-Year Case-Study. Gen Med (Los Angeles) 4: 254. doi:10.4172/2327-5146.1000254

Page 3 of 3

statistically significant correlation between the results obtained by people with PD in the posturographic test and UPDRS [17].

\section{Conclusions}

An increase in postural sway in both the sagittal and frontal planes was observed in patients after direct double-sided DBS implantation and pharmacological treatment. During the six-month observation period after combined DBS and physiotherapy, improvement occurred in the analyzed parameters. Computer testing with the usage of the balance platform is a repeatable method that can be successfully applied in the assessment of movement disorders and the analysis of the results of pharmacological treatment, DBS or the physiotherapy of patients with PD.

\section{References}

1. Bergman H, Deuschl G (2002) Pathophysiology of Parkinson's disease from clinical neurology to basic neuroscience and back. Mov Disord 17: S28-40.

2. Friedman A (2005) In. Epidemiology Friedman A (ed.). Parkinson's disease, the mechanisms, diagnosis, treatment. Lublin: Publisher Czelej $1-5$.

3. Adkin AL, Frank JS, Jog MS (2003) Fear of falling and postural control in Parkinson's disease. Mov Disord 18: 496-502.

4. Winter DA (1995) Human balance and posture control during standing and walking. Gait \& Posture 3: 193-214.

5. Błaszczyk J (2004) Biomechanika kliniczna. Warszawa 192-232.

6. Mancini M, Horak FB, Zampieri C, Carlson-Kuhta P, Nutt JG, et al. (2011) Trunk accelerometry reveals postural instability in untreated Parkinson's disease. Parkinsonism Relat Disord 17: 557-562.
7. Carpenter MG, Allum JH, Honegger F, Adkin AL, Bloem BR (2004) Postural abnormalities to multidirectional stance perturbations in Parkinson's disease. J Neurol Neurosurg Psychiatry 75: 1245-1254.

8. Kim SD, Allen NE, Canning CG, Fung VS (2013) Postural instability in patients with Parkinson's disease. Epidemiology, pathophysiology and management. CNS Drugs 27: 97-112.

9. Fahn S, Jankovic J, Halett M (2011) Principles and practise of movement disorders. Elsevier Sanders, pp: 20-156.

10. Kringelbach ML, Jenkinson N, Owen SL, Aziz TZ (2007) Translational principles of deep brain stimulation. Nat Rev Neurosci 8: 623-635.

11. Schüpbach WM, Chastan N, Welter ML, Houeto JL, Mesnage V, et al. (2005) Stimulation of the subthalamic nucleus in Parkinson's disease: a 5 year follow up. J Neurol Neurosurg Psychiatry 76: 1640-1644.

12. Mraz M, Cembala A, Chamela-Bilińska D, Mraz M (2001) Postural stability in patients with Parkinson's disease determined on the basis of posturographic. Physiotherapy 9: 23-25.

13. Czechowicz B, Boczarska-Jedynak M, Opala G, Slomka K (2011) The influence of visual control on postural stability in Parkinson disease. Neurol Neurochir Pol 45: 132-139.

14. Rocchi L, Chiari L, Horak B (2002) Effects of deep brain stimulation and levodopa on postural sway in Parkinson's disease. J Neurol Neurosurg Psychiatry 73: 267-274.

15. Deep-Brain Stimulation for Parkinson's Disease Study Group (2001) Deep-brain stimulation of the subthalamic nucleus or the pars interna of the globus pallidus in Parkinson's disease. N Engl J Med 345: 956-963.

16. Fukunaga JY, Quitschal RM, Doná F, Ferraz HB, Ganança MM, et al. (2014) Postural control in Parkinson's disease. Braz J Otorhinolaryngol 80: 508-514.

17. Matinolli M, Korpelainen JT, Korpelainen R, Sotaniemi KA, Virranniemi M, et al. (2007) Postural sway and falls in Parkinson's disease: a regression approach. Mov Disord 22: 1927-1935. 